\title{
Effect of MFD Viscosity on the Onset of Ferromagnetic Fluid Layer Heated from Below and Cooled from Above with Constant Heat Flux
}

\author{
C. E. Nanjundappa ${ }^{1}$, I. S. Shivakumara ${ }^{2}$, K. Srikumar ${ }^{3}$ \\ ${ }^{1}$ Department of Mathematics, Dr. Ambedkar Institute of Technology, Bangalore -560 056,INDIA, e mail: cenanju@hotmail.com \\ ${ }^{2}$ UGC-Centre for Advanced Studies in Fluid Mechanics, Department of Mathematics, Bangalore University, \\ Bangalore - 560 001, INDIA, e mail: shivakumarais@gmail.com \\ ${ }^{3}$ Department of Mathematics, C M R Institute of Technology, Bangalore -560 037, INDIA,
}

\begin{abstract}
The effect of magnetic field dependent (MFD) viscosity on the onset of convection in a ferromagnetic fluid layer heated from below and cooled from above in the presence of vertical magnetic field is investigated theoretically. The bounding surfaces are considered to be either rigid-ferromagnetic or stress free with constant heat flux conditions. The resulting eigenvalue problem is solved using the Galerkin technique and also by regular perturbation technique. It is found that increase in MFD viscosity and decrease in magnetic number is to delay the onset of ferroconvection, while the nonlinearity of fluid magnetization has no influence on the stability of the system.
\end{abstract}

Keywords: ferromagnetic fluid, constant heat flux, Galerkin technique, MFD viscosity

\section{INTRODUCTION}

$\mathrm{F}$ ERROFLUIDS are stable colloidal suspensions consisting of single-domain magnetic particles coated with a surfactant and immersed in a carrier fluid. The particles are commonly magnetite and of order $10 \mathrm{~nm}$ in diameter, while the carrier liquid is typically an oil or water base. Ferrofluids are of particular interest since there are no known natural occurrences of these fluids, and in distinction to magnetohydrodynamics the flow phenomena occur without the need for electrical current, and thus in the absence of corresponding Lorentz forces. Thereafter, ferrofluids of various kinds have been developed and their application to engineering uses attempted. Presently, magnetic fluids are in wide use in seals, bearings, magnetostatic support, jet printers, separation of nonmagnetic particles, flow control and drag reduction, dampers, actuators, sensors, transducers, and medical applications. An authoritative introduction to this fascinating subject is amply provided in the literature [1], [2]. Thus, ferrofluids have received much attention in the scientific community.

The magnetization of ferrofluids depends on the magnetic field, temperature, and density. Hence, any variations of these quantities induce change of body force distribution in the fluid and eventually give rise to convection in ferrofluids in the presence of a gradient of magnetic field. There have been numerous studies on thermal convection in a ferrofluid layer called ferroconvection analogous to Rayleigh-Benard convection in ordinary viscous fluids. The theory of convective instability in a horizontal layer of ferrofluid began with [3] and extensively continued over the years [4]-[10] and references therein. A variety of velocity and temperature boundary conditions on the onset of ferroconvection in an initially quiescent ferrofluid layer in the presence of a uniform magnetic field has been investigated recently [11]. Besides, studies have been made to understand the effect of different parameters on thermomagnetic convection of ferrofluids in a cylindrical geometry using the modern Taguchi technique [12] and heat transfer phenomena in a kerosene based ferrofluid in two cylinders with different dimensions using computation fluid dynamics technique [13]. Investigations have also been carried out to analyze magnetic convection of ferrofluids in enclosures [14]-[16].

Thermal convection in ferromagnetic fluids is gaining much importance due to astounding physical properties. One such property is viscosity of the ferromagnetic fluid. Fluids with ferromagnetic properties may be formed by colloidal suspension of solid magnetic particles such as magnetite in a parent liquid. The viscosity of the magnetic fluid is predicted by dimensional analysis to be a function of the ratio of hydrodynamic stress to magnetic stress [17]. The effect of a homogeneous magnetic field on the viscosity of a fluid with solid particles possessing intrinsic magnetic moments has been investigated [18]. The effect of magnetic field dependent (MFD) viscosity on the onset of ferroconvection in a rotating medium [19], with or without dust particles [20] and the nonlinear stability analysis [21] have been examined. All these studies are dealt with isothermal boundary conditions.

The present study deals with the effect of MFD viscosity on the onset of convection in a ferromagnetic fluid layer in the presence of a uniform vertical magnetic field for different combinations of velocity boundary conditions with prescribed heat flux at the boundaries. Such a study is useful in the proper design of numerous devices and processes such as levitation accelerometers magneticfluids seals, direct energy converters and viscous dampers [17]. The resulting eigenvalue problem is solved using the Galerkin technique and also regular perturbation technique for both boundaries rigid (rigid-rigid), both boundaries free (free-free), and lower boundary rigid and upper boundary free (rigid - free) boundary combinations.

To achieve the above objectives, the paper is organized as follows. Section 2 is devoted to mathematical formulation. The method of solution is discussed in Section 3. In Section 4, the numerical results presented are discussed and some important conclusions follow in Section 5. 


\section{MATHEMATICAL FORMULATION}

The system under consideration is an initially quiescent horizontal ferrofluid layer of characteristic thickness $d$ in the presence of an applied magnetic field $H_{O}$ in the vertical direction. The lower and the upper boundaries are maintained at constant temperature $T_{0}$ and $T_{1}\left(<T_{0}\right)$ respectively, and thus constant temperature difference $\Delta T\left(=T_{1}-T_{0}\right)$ is maintained between the boundaries. A Cartesian co-ordinate system $(x, y, z)$ is used with the origin at the bottom of the layer and $z$-axis is directed vertically upward. Gravity acts in the negative $\mathrm{z}$-direction, $\vec{g}=-g \hat{k}$, where $\hat{k}$ is the unit vector in the z-direction. The fluid is assumed to be incompressible having variable viscosity, given by $\eta=\eta_{0}(1+\vec{\delta} \cdot \vec{B})$, where $\vec{\delta}$ is the variation coefficient of magnetic field dependent viscosity and is considered to be isotropic [19], $\eta_{0}$ is taken as viscosity of the fluid when the applied magnetic field is absent and $\vec{B}=\left(B_{x}, B_{y}, B_{z}\right)$ is the magnetic induction. Experimentally, it has been demonstrated that the magnetic viscosity has got exponential variation, with respect to magnetic field [22]. As a first approximation for small field variation, linear variation of magnetic viscosity has been used. The equations governing the flow of an incompressible ferrofluid are [1,2] :

$$
\begin{aligned}
& \nabla \cdot \vec{q}=0 \\
& \rho_{0}\left[\frac{\partial \vec{q}}{\partial t}+(\vec{q} \cdot \nabla) \vec{q}\right]=-\nabla \mathrm{p}+\rho \vec{g} \\
& +2 \nabla \cdot[\eta \underline{\underline{D}}]+\mu_{0}(\vec{M} \cdot \nabla) \vec{H}, \\
& {\left[\rho_{0} C_{V, H}-\mu_{0} \vec{H} \cdot\left(\frac{\partial \vec{M}}{\partial T}\right)_{V, H}\right] \frac{D T}{D t}} \\
& +\mu_{0} T\left(\frac{\partial \vec{M}}{\partial T}\right)_{V, H} \cdot \frac{D \vec{H}}{D t}=k_{t} \nabla^{2} T \\
& \rho=\rho_{0}\left[1-\alpha_{t}\left(T-T_{0}\right)\right], \\
& \nabla \cdot \vec{B}=0, \nabla \times \vec{H}=0 \text { or } \vec{H}=\nabla \varphi, \\
& \vec{B}=\mu_{0}(\vec{M}+\vec{H}), \\
& \vec{M}=\frac{\vec{H}}{H} M(H, T), \\
& M=M_{0}+\chi\left(H-H_{0}\right)-K\left(T-T_{0}\right) .
\end{aligned}
$$

Here, $\vec{q}=(u, v, w)$ is the velocity vector, $\vec{M}$ the magnetization, $\vec{H}$ the magnetic intensity of the fluid, $p$ the pressure, $t$ the time, $\underline{\underline{D}}=\left[\nabla \vec{q}+(\nabla \vec{q})^{T}\right] / 2$ the rate of strain tensor, $\rho$ the fluid density, $\rho_{0}$ the density at $T=T_{0}, \mu_{0}$ the magnetic permeability of vacuum, $k_{t}$ the thermal conductivity of the fluid, $C_{V, H}$ the specific heat at constant volume and magnetic field, $\alpha_{t}$ the thermal expansion coefficient, $\varphi$ the magnetic potential, $\chi=(\partial M / \partial H)_{H_{0}}, T_{0}$ the magnetic susceptibility, $K=-(\partial M / \partial T)_{H_{0}}, T_{0}$ the pyromagnetic coefficient , $\nabla^{2}=\partial^{2} / \partial x^{2}+\partial^{2} / \partial y^{2}+\partial^{2} / \partial z^{2}$ the Laplacian operator and $M_{0}=M\left(H_{0}, T_{0}\right)$.

It is clear that there exists the following solution for the basic state:

$$
\begin{aligned}
& \vec{q}_{b}=0, \\
& p_{b}(z)=p_{0}-\rho_{0} g z-\frac{1}{2} \rho_{0} \alpha_{t} g \beta z^{2} \\
& -\frac{\mu_{0} M_{0} \kappa \beta z}{(1+\chi)}-\frac{\mu_{0} \kappa^{2} \beta^{2} z^{2}}{2(1+\chi)^{2}}, \\
& T_{b}(z)=T_{0}-\beta z, \\
& \vec{H}_{b}(z)=\left[H_{0}-\frac{K \beta z}{(1+\chi)}\right] \hat{k}, \\
& \vec{M}_{b}(z)=\left[M_{0}+\frac{K \beta z}{(1+\chi)}\right] \hat{k}
\end{aligned}
$$

where $\beta=\Delta T / d$ is the temperature gradient and the subscript $b$ denotes the basic state. To investigate the conditions under which the quiescent solution is stable against small disturbances, we consider a perturbed state such that

$$
\begin{aligned}
& \vec{q}=\vec{q}^{\prime}, \\
& p=p_{b}(z)+p^{\prime}, \\
& \eta=\eta_{b}(z)+\eta^{\prime}, \\
& T=T_{b}(z)+T^{\prime}, \\
& \vec{H}=\vec{H}_{b}(z)+\vec{H}^{\prime}, \\
& \vec{M}=\vec{M}_{b}(z)+\vec{M}^{\prime}
\end{aligned}
$$

where $\overrightarrow{\mathrm{q}}^{\prime}, \mathrm{p}^{\prime}, \eta^{\prime}, \mathrm{T}^{\prime}, \overrightarrow{\mathrm{H}}^{\prime}$ and $\overrightarrow{\mathrm{M}}^{\prime}$ are perturbed variables and are assumed to be small.

Substituting Eq. (10) into Eqs. (6) and (7), and using Eq.(5), we obtain (after dropping the primes)

$$
\begin{aligned}
& H_{x}+M_{x}=\left(1+\frac{M_{0}}{H_{0}}\right) H_{x}, \\
& H_{y}+M_{y}=\left(1+\frac{M_{0}}{H_{0}}\right) H_{y}, \\
& H_{z}+M_{z}=(1+\chi) H_{z}-K T .
\end{aligned}
$$

Again substituting Eq. (10) into the momentum Eq. (2), linearizing, eliminating the pressure term by operating curl twice and using Eq. (11) the z-component of the resulting equation can be obtained as (after dropping the primes) 


$$
\begin{array}{r}
\left(\rho_{0} \frac{\partial}{\partial t}-\eta_{b} \nabla^{2}\right) \nabla^{2} w=-\mu_{0} K \beta \frac{\partial}{\partial z}\left(\nabla_{h}^{2} \varphi\right) \\
+\frac{\mu_{0} K^{2} \beta}{1+\chi} \nabla_{h}^{2} T+\rho_{0} \alpha_{t} g \nabla_{h}^{2} T
\end{array}
$$

where $\nabla_{h}^{2}=\partial^{2} / \partial x^{2}+\partial^{2} / \partial y^{2}$ is the horizontal Laplacian operator and $\eta_{b}=\eta_{0}\left[1+\delta \mu_{0}\left(M_{0}+H_{0}\right)\right]$.

The energy Eq. (3), after using Eq. (10) and linearizing, takes the form (after dropping the primes)

$$
\begin{aligned}
& \rho_{0} C_{0} \frac{\partial T}{\partial t}-\mu_{0} T_{0} K \frac{\partial}{\partial t}\left(\frac{\partial \varphi}{\partial z}\right)= \\
& {\left[\rho_{0} C_{0}-\frac{\mu_{0} T_{0} K^{2}}{1+\chi}\right] w \beta+k_{1} \nabla^{2} T}
\end{aligned}
$$

where $\rho_{0} C_{0}=\rho_{0} C_{V, H}+\mu_{0} H_{0} K$. Equations 5(a, b), after substituting Eq. (10) and using Eq. (11), may be written as

$$
\begin{aligned}
\left(1+\frac{M_{0}}{H_{0}}\right) \nabla_{h}^{2} \varphi+(1+\chi) & \frac{\partial^{2} \varphi}{\partial z^{2}} \\
& -K \frac{\partial T}{\partial z}=0 .
\end{aligned}
$$

Since the principle of exchange of stability is valid [3], the normal mode expansion of the dependent variables is assumed in the form

$$
\{w, T, \varphi\}=\{W(z), \Theta(z), \Phi(z)\} \exp [i(\ell x+m y)]
$$

where $\ell$ and $m$ are wave numbers in the $x$ and $y$ directions, respectively. On substituting Eq. (15) into Eqs. (12) - (14) and non-dimesionalizing the variables by setting

$$
\begin{aligned}
& \left(x^{*}, y^{*}, z^{*}\right)=\left(\frac{x}{d}, \frac{y}{d}, \frac{z}{d}\right), \\
& W^{*}=\frac{d}{v} W, \\
& \Theta^{*}=\frac{\kappa}{\beta v d} \Theta, \\
& \Phi^{*}=\frac{1+\chi) \kappa}{\mathrm{K} \beta v d^{2}} \Phi \text { and } \\
& \delta^{*}=\mu_{0} H_{0}(1+\chi) \delta
\end{aligned}
$$

where $v=\eta_{0} / \rho_{0}$ is the kinematic viscosity and $\kappa=k_{t} / \rho_{0} C_{0}$ is the effective thermal diffusivity, we obtain

$$
\begin{gathered}
(1+\delta)\left(D^{2}-a^{2}\right)^{2} W+a^{2} R M_{1} D \Phi- \\
a^{2} R\left(1+M_{1}\right) \Theta=0 \\
\left(D^{2}-a^{2}\right) \Theta+\left(1-M_{2}\right) W=0 \\
\left(D^{2}-a^{2} M_{3}\right) \Phi-D \Theta=0 .
\end{gathered}
$$

Here, $D=d / d z$ is the differential operator, $a=\sqrt{\ell^{2}+m^{2}}$ is the overall horizontal wavenumber, $R=\alpha_{t} g \beta d^{4} / \nu \kappa$ is the thermal Rayleigh number, $M_{1}=\mu_{0} K^{2} \beta /(1+\chi) \alpha_{t} \rho_{0} g$ is the magnetic number, $M_{2}=\mu_{0} T_{0} K^{2} / \rho_{0} C_{0}(1+\chi) \quad$ is the magnetic parameter and $M_{3}=\left(1+M_{0} / H_{0}\right) /(1+\chi)$ is the measure of nonlinearity of magnetization. The typical value of $M_{2}$ for magnetic fluids with different carrier liquids turns out to be of the order of $10^{-6}$ and hence its effect is neglected as compared to unity.

The bounding surfaces (rigid or free) of the ferrofluid layer are considered to be ferromagnetic and insulated to temperature perturbations. Thus, on the rigid boundary, $W=D W=\Phi=D \Theta=0$ and on the stress-free boundary $W=D^{2} W=D \Phi=D \Theta=0$.

\section{METHOD OF SOLUTION}

Equations (17) - (19) together with the corresponding boundary conditions constitute an eigenvalue problem with $R$ as an eigenvalue. The method of solution is described in the following sub-sections.

\subsection{Solution by galerkin technique}

The Galerkin method is used to solve this problem as explained [23]. In this method, the test (weighted) functions are the same as the base (trial) functions. Accordingly, $W, \Theta$ and $\Phi$ are written as

$$
\begin{aligned}
& W=\sum_{i=1}^{n} A_{i} W_{i}(z), \\
& \Theta(z)=\sum_{i=1}^{n} C_{i} \Theta_{i}(z), \\
& \Phi(z)=\sum_{i=1}^{n} D_{i} \Phi_{i}(z)
\end{aligned}
$$

where $A_{i}, C_{i}$ and $D_{i}$ are the unknown constants to be determined. The base functions $W_{i}(z), \Theta_{i}(z)$ and $\Phi_{i}(z)$ are generally chosen such that they satisfy the corresponding boundary conditions but not the differential equations.

For rigid-rigid, rigid-free and free-free boundaries the base the base functions are, respectively

$$
\begin{aligned}
& W_{i}=\left(z^{4}-2 z^{3}+z^{2}\right) T_{i-1}^{*}, \\
& \Theta_{i}=z^{2}(1-2 z / 3) T_{i-1}^{*}, \\
& \Phi_{i}=\left(z^{2}-z\right)(z-2) T_{i-1}^{*} . \\
& W_{i}=\left(2 z^{4}-5 z^{3}+3 z^{2}\right) T_{i-1}^{*}, \\
& \Phi_{i}=z^{2}(1-2 z / 3) T_{i-1}^{*}, \\
& \Phi_{i}=z^{2}(1-2 z / 3) T_{i-1}^{*} .
\end{aligned}
$$




$$
\begin{aligned}
& W_{i}=\left(z^{4}-2 z^{3}+z\right) T_{i-1}^{*}, \\
& \Theta_{i}=z^{2}(1-2 z / 3) T_{i-1}^{*}, \\
& \Phi_{i}=z^{2}(1-2 z / 3) T_{i-1}^{*}
\end{aligned}
$$

where $T_{i}^{*}{ }^{*} s$ are the modified Chebyshev polynomials. The above trial functions satisfy all the boundary conditions. Multiplying Eq. (17) by $W_{i}(z)$, Eq. (18) by $\Theta_{i}(z)$ and Eq. (19) by $\Phi_{i}(z)$; performing the integration by parts with respect to $\mathrm{z}$ between $\mathrm{z}=0$ and $\mathrm{z}=1$ and using the boundary conditions, we obtain a system of linear homogeneous algebraic equations in $A_{i}, C_{i}$ and $D_{i}$. A non-trivial solution to these equations leads to a relation involving the physical parameters $R, \delta, M_{1}, M_{3}$ and the wave number $a$ in the form $f\left(R, \delta, M_{1}, M_{3}, a\right)=0$. The critical value of $R$ (i.e., $R_{c}$ ) is determined numerically with respect to $a$ for different values of $\delta, M_{1}$ and $M_{3}$.

\subsection{Solution by regular perturbation technique}

Since the critical wave number is negligibly small when the boundaries are insulated to temperature perturbations (i.e., $D \Theta=0$ at $z=0,1$ ), the eigenvalue problem is also solved analytically using regular perturbation technique with wave number $a$ as a perturbation parameter. Accordingly, the variables $W, \Theta$ and $\Phi$ are expanded in powers of $a^{2}$ as

$$
(W, \Theta, \Phi)=\left(W_{0}, \Theta_{0}, \Phi_{0}\right)+a^{2}\left(W_{1}, \Theta_{1}, \Phi_{1}\right)+\cdots
$$

Substituting Eq. (24) into Eqs.(17) - (19) as well as in the boundary conditions, the terms of different orders are collected and solved. The solution to the zero-th order equations for rigid-rigid and rigid- free boundaries is found to be $W_{0}=0, \Theta_{0}=1$ and $\Phi_{0}=0$, while the solution for freefree boundaries is given by $W_{0}=0, \Theta_{0}=1$ and $\Phi_{0}=1$. The general solution for velocity at the first order for rigid-rigid, rigid-free and free-free boundary conditions, respectively is given by

$$
\begin{aligned}
& W_{1}=\frac{R\left(1+M_{1}\right)\left(z^{4}-2 z^{3}+z^{2}\right)}{24(1+\delta)}, \\
& W_{1}=\frac{R\left(1+M_{1}\right)\left(2 z^{4}-5 z^{3}+3 z^{2}\right)}{24(1+\delta)}
\end{aligned}
$$

and

$$
W_{1}=\frac{R\left(1+M_{1}\right)\left(z^{4}-2 z^{3}+z\right)}{24(1+\delta)} .
$$

An expression for the critical Rayleigh number for rigid-rigid, rigid-free and free-free boundary conditions is obtained from the solvability condition

$$
1=\int_{0}^{1} W_{1} d z
$$

respectively, in the form

$$
\begin{aligned}
& R_{c}=\frac{720(1+\delta)}{\left(1+M_{1}\right)}, \\
& R_{c}=\frac{320(1+\delta)}{\left(1+M_{1}\right)}
\end{aligned}
$$

and

$$
R_{c}=\frac{310(1+\delta)}{\left(1+M_{1}\right)}
$$

It is interesting to check the above relations for some special cases. Letting $\delta=0$, Eqs. (27a,b,c) respectively, reduce to

$$
\begin{aligned}
& R_{c}=\frac{720}{\left(1+M_{1}\right)}, \\
& R_{c}=\frac{320}{\left(1+M_{1}\right)}, \\
& R_{c}=\frac{120}{\left(1+M_{1}\right)}
\end{aligned}
$$

and thus recovering the results for the case of constant viscosity [11]. When $M_{1}=0$ (i.e. ordinary viscous fluid), Eqs. (28a,b,c) reduce to the critical Rayleigh numbers of $R_{c}=720,320$ and 120 which are the known exact values for the rigid-rigid, rigid-free and free-free boundaries, respectively. From Eqs. $(27 \mathrm{a}, \mathrm{b}, \mathrm{c})$, it is interesting to note that the nonlinearity of fluid magnetization (i.e., $M_{3}$ ) has no effect on the onset of convection; a result which is revealed by numerical computations carried out in the previous section. Since at the onset of convection $a_{c}=0$ (very large wave length), one would expect that $M_{3}$ has no effect on the stability of the system (see Eq.19). The numerically computed values of $R_{c}$ for different values of $\delta$ and $M_{1}$ are compared in Table 1 with those obtained using regular perturbation technique. We note that the results obtained from simple regular perturbation technique coincide exactly with those obtained from time consuming numerical methods and thus provide a justification for the results obtained analytically.

\section{Results AND Discussion}

The effect of MFD viscosity $\delta$ on the onset of convection in a ferrofluid layer in the presence of a uniform vertical magnetic field is studied. The boundaries are considered to be insulated to temperature perturbations and the resulting eigenvalue problem is solved numerically using the Galerkin technique as well as analytically by employing regular perturbation technique with wave number as a perturbation parameter. The critical eigenvalue $R_{c}$ obtained numerically and analytically for different values of $M_{1}, \delta$ and for different boundary conditions are presented in Table 1. Since the rigid boundaries suppress the perturbations to a greater extent compared to the free boundaries, we note that the critical Rayleigh number $R_{c}$ for rigid-rigid boundaries is the greatest, followed by rigid-free boundaries and the least 
for free-free boundaries. The table also predicts the effect of $\delta$, which represents the effect of viscosity variation with magnetic field, on the criterion for the onset of convection. We note that increase in $\delta$, though the effect is not so significant, has a stabilizing effect on the system. That is, the effect of increase in the value of $\delta$ is to delay the onset of ferroconvection. An increase in the value of $M_{1}$ is to decrease the value of $R_{c}$ and thus its effect is to augment ferroconvection due to an increase in the magnetic force.
The vertical velocity eigenfunctions $W(z)$ for different boundary combinations are presented in Fig.1 and 2 for different values of $\delta$ and $M_{1}$, respectively. As can be seen, increase in the value of MFD viscosity $\delta$ (see Fig.1) is to inhibit the ferrofluid flow and hence its effect is to delay the onset of ferroconvection. However, increase in the value of $M_{1}$ (see Fig.2) is to accelerate the ferrofluid flow and hence its effect is to hasten the onset of ferroconvection.

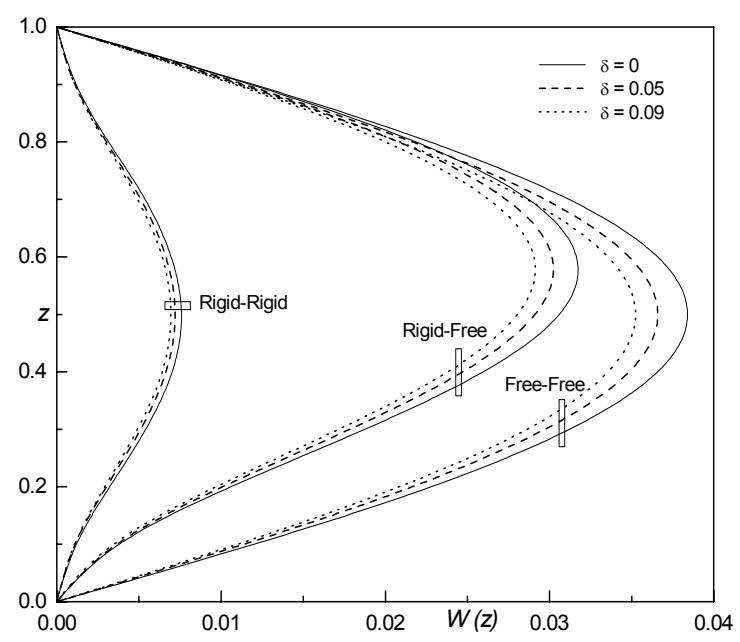

Fig.1 Vertical velocity eigenfunction for different values of $\delta$ when $M_{1}=2$

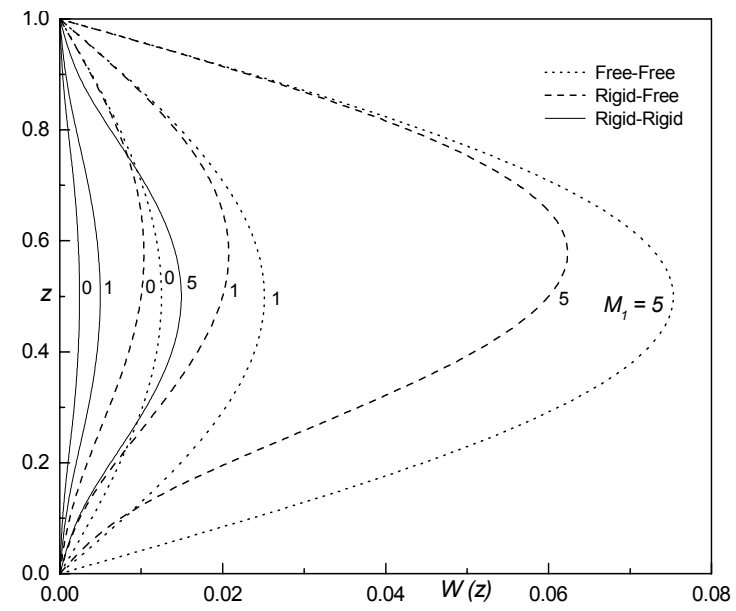

Fig.2 Vertical velocity eigenfunction for different values of $M_{1}$ when $\delta=0.02$

Table 1. Critical Rayleigh number $R_{c}$ for various values of $M_{1}, \delta$ and for different boundaries

\begin{tabular}{|c|ccc|c|c|c|c|ccc|}
\hline \multirow{2}{*}{$M_{1}$} & \multicolumn{4}{|c|}{ Rigid-Rigid boundaries } & \multicolumn{4}{c|}{ Rigid-Free boundaries } & \multicolumn{3}{c|}{ Free-Free boundaries } \\
& $\delta=0$ & $\delta=0.05$ & $\delta=0.09$ & $\delta=0$ & $\delta=0.05$ & $\delta=0.09$ & $\delta=0$ & $\delta=0.05$ & $\delta=0.09$ \\
& $R_{c}$ & $R_{c}$ & $R_{c}$ & $R_{c}$ & $R_{c}$ & $R_{c}$ & $R_{c}$ & $R_{c}$ & $R_{c}$ \\
\hline 0 & 720 & 756 & 784.8 & 320 & 336 & 348.8 & 120 & 126 & 130.8 \\
1 & 360 & 378 & 392.4 & 160 & 168 & 174.4 & 60 & 63 & 65.4 \\
2 & 240 & 252 & 261.6 & 106.66 & 112 & 116.26 & 40 & 42 & 43.6 \\
3 & 180 & 189 & 196.2 & 80 & 84 & 87.2 & 30 & 31.5 & 32.7 \\
4 & 144 & 151.2 & 156.9 & 64 & 67.2 & 69.76 & 24 & 25.2 & 26.16 \\
5 & 120 & 126 & 130.8 & 53.33 & 56 & 58.13 & 20 & 21 & 21.8 \\
\hline
\end{tabular}




\section{CONCLUSIONS}

From the foregoing study, it is observed that an increase in the value of $\delta$ and decrease in $M_{1}$ is to stabilize the ferrofluid motion against the convection. In contrast to the isothermal boundaries case, the nonlinearity of fluid magnetization $M_{3}$ is found to have no effect on the stability of the system. The critical eigenvalues obtained from the combination of analytical and numerical techniques complement very closely with Each other and note that

$$
\left(R_{c}\right)_{\text {rigid-rigid }}>\left(R_{c}\right)_{\text {rigid-free }}>\left(R_{c}\right)_{\text {free-free }} .
$$

\section{ACKNOWLEDGEMENTS}

The work reported in this paper was supported by UGC under CAS Program, Department of Mathematics, Bangalore University. The authors (CEN) and (K.S) wish to thank the Management and Principal of Dr. Ambedkar Institute of Technology, and C M R Institute of Engineering and Technology, Bangalore, respectively, for their encouragement

\section{REFERENCES}

[1] Rosensweig, R.E. (1985). Ferrohydrodynamics. Cambridge: Cambridge University Press.

[2] Bashtovoy, V.G., Berkovsky, B.N., Vislovich, A.N. (1988). Introduction to Thermomechanics of Magnetic Fluids. Washington: Hemisphere Publishing Corp.

[3] Finlayson, B.A. (1970). Convective instability of ferromagnetic fluids. Journal of Fluid Mechanics, 40, 753-767.

[4] Odenbach, S. (2003). Magneticfluids-suspensionsof magnetic dipoles and their mantic controls. Journal of Physics, 15, 1497-1508.

[5] Lalas, D.P., Carmi, S. (1971). Thermoconvective stability of ferrofluids. Physics of Fluids, 14, 436-437.

[6] Shliomis, M.I. (1974). Magneticfluids. Soviet Physics Uspekhi, 17, 153-169.

[7] Gotoh, K., Yamada, M. (1982). Thermal convection in a horizontal layer of magneticfluids. Journal of Physical Society of Japan, 51, 3042-3048.

[8] Schwab, L., Hildebrandt, U., Stierstadt, K. (1983). Magnetic Bénard convection. Journal of Magnetism and Magnetic Materials, 39, 113-114.

[9] Stiles, P.J., Kagan, M.J. (1990). Thermoconvective instability of a ferrofluid in a strong magnetic field. Journal of Colloid and Interface Science, 134, 435-449.

[10] Mahjan, S., Mahjan, A. (2008). Nonlinear stability analysis for magnetized ferrofluid heated from below. Proceedings of Royal Society of London A:
Mathematical Physical \& Engineering Science, 464, 83-98.

[11] Nanjundappa, C.E., Shivakumara, I.S. (2008). Effect of velocity and temperature boundary conditions on convective instability in a ferrofluid layer. Journal of Heat Transfer, 130,104502-1-1045021-5.

[12] Jafari, A., Tynjälä, T., Mousavi, S.M., Sarkomaa, P. (2008). CFD simulation and evaluation of controllable parameters effect on thermomagnetic convection in ferrofluids using Taguchi technique. Computers and Fluids, 37 (10), 1344-1353.

[13] Jafari, A., Tynjälä, T., Mousavi, S.M., Sarkomaa, P. (2008). Simulation of heat transfer in a ferrofluid using computational fluid dynamics technique. International Journal of Heat and Fluid Flow, 29 (4), 1197-1202.

[14] Jue, T.-C. (2006). Analysis of combined thermal and magnetic convection ferrofluid flow in a cavity. International Communications in Heat and Mass Transfer, 33 (7), 846-852.

[15] Tangthieng, C., Finlayson, B.A., Maulbetsch, J., Cader, T. (1999). Heat transfer enhancement in ferrofluids subjected to steady magnetic fields. Journal of Magnetism and Magnetic Materials, 201 (1-3), 252-255.

[16] Snyder, S.M., Cader, T., Finlayson, B.A. (2003). Finite element model of magnetoconvection of a Ferrofluid. Journal of Magnetism and Magnetic Materials 262 (2), 269-279.

[17] Rosenwieg, R.E., Kaiser, R., Miskolczy, G. (1969). Viscosity of magnetic fluid in a magnetic field. Journal of Colloid and Interface Science 29 (4), 680-686.

[18] Shliomis, M.I. (1972). Effect of viscosity of magnetic suspensions. Soviet Physics JETP, 34, 1291-1294.

[19] Vaidyanathan, G., Sekar, R., Ramanathan, A. (2002). Effect of magnetic field dependent viscosity on ferroconvection in rotating medium. Indian Journal of Pure and Applied Physics, 40, 159-165.

[20] Sunil, Sharma, A., Shandil, R.G. (2008). Effect of magnetic field dependent viscosity on ferroconvection in the presence of dust particles. Journal of Applied Mathematics and Computing, 27, 7-22.

[21] Sunil, Sharma, P. , Mahajan, A. (2008). A nonlinear stability analysis for thermoconvective magnetized ferrofluid with magnetic field dependent viscosity. International Communications in Heat and Mass Transfer, 35 (10), 1281-1287.

[22] Rosenwieg, R.E. (1966). Bouyancy and stable levitation of a magnetic body immersed in a magnetizable fluid. Nature, 210, 613-614.

[23] Finlayson, B.A. (1972). Method of Weighted Residuals and Variational Principles. Academic Press. 